\title{
Nonlinear Interaction Imaging and Spectroscopy in Scanning Probe Microscopy
}

\author{
Stephen Jesse
}

The Center for Nanophase Materials Sciences, Oak Ridge National Laboratory, PO Box 2008, Oak Ridge, TN 37831-2008

All information about material properties acquired through scanning probe microscopy is derived from the measurements of interactions between the tip and the sample. Nearly every form of scanning probe technique developed to date is based on measuring and recording only the linear aspects of this interaction and disregarding nonlinear components. Though much can be derived from looking at only the linear interactions, there is perhaps more important information hidden within the nonlinear ones such as local field gradients, indentation forces, hysteresis associated with anelastic and ferroic effects etc.. Of particular value are the short range forces that are generated when the tip is closest to the surface. We have developed an atomic force microscope control and acquisition scheme that can determine both the linear and non-linear components of tip-surface interaction of a tip in motion, at resonance, and thereby extract both the usual metrics of tip motion, such as amplitude, resonance, and dissipation, as well as the force-distance curves for arbitrary tipsurface interactions. This method is based on performing linear operations on measured data and systematically extracting the non-linear components to reconstruct the potential well.

We base this technique on the recognition that, according to the equation of motion for a particle within a potential well: $\quad m \cdot \ddot{x}(t)+b \cdot \dot{x}(t)+[k+p(x(t))] \cdot x=h(t)$

(where $x$ is position, $m, b$, and $k$ are effective mass, damping, and spring constants, $h(t)$ is the externally applied force, and $p(x(t))$ is the non-linear contribution to the force-distance curve) in the absence of any non-linear contributions, all points in the 3-D phase-space trajectory must lie within a plane (figure 1a). And, by extension, deviations from this plane are due to non-linearities in the force-distance curve $(p(x))$ as seen in figure 1b. Straightforward methods for extracting $p(x)$ are used. We will demonstrate this approach for modeled data, and experimental data including force-distance curves, tapping mode, piezo-response force microscopy (contact mode), and magnetic force microscopy (non-contact mode). The methodology developed here can be used as a general protocol applicable to the measurement and analysis of wide variety of oscillating systems. This capability allows for the implementation of dynamic spectroscopies of local bias- and temperature-induced phase transitions, in which the changes in dynamic cantilever response as a function of external parameter provide information about local nanoscale functionalities.

\section{Reference}

[1] Research at Oak Ridge National Laboratory's Center for Nanophase Materials Sciences was sponsored by the Scientific User Facilities Division, Office of Basic Energy Sciences, U.S. Department of Energy. 
FIG. 1 .

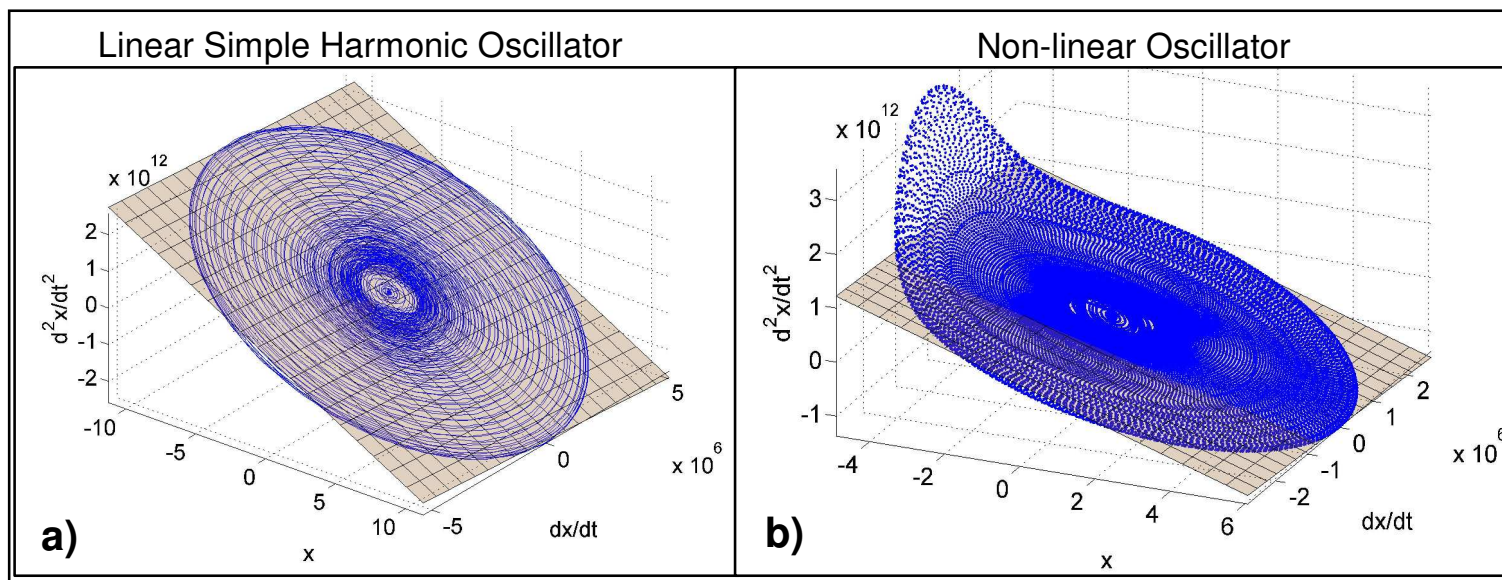

Figure 1: Plots of position $(x(t))$ vs. velocity $(\dot{x}(t))$ vs. acceleration $(\ddot{x}(t))$ of the modeled response of the perfectly linear simple harmonic oscillator and for oscillations within the Leonard-Jones potential along with their best-fit planes. For the linear case, (a) all data points within the plot lie in a plane. Therefore, it is possible to directly extract all of the dynamic parameters (mass, damping, and spring constant) directly from the plane fit. In the case of a non-linear oscillation, the best fit plane gives the mass, damping, and the linear component of the spring constant as well, but deviations from linearity are clear and can be extracted to reconstruct the anharmonic potential well.

FIG. 2.
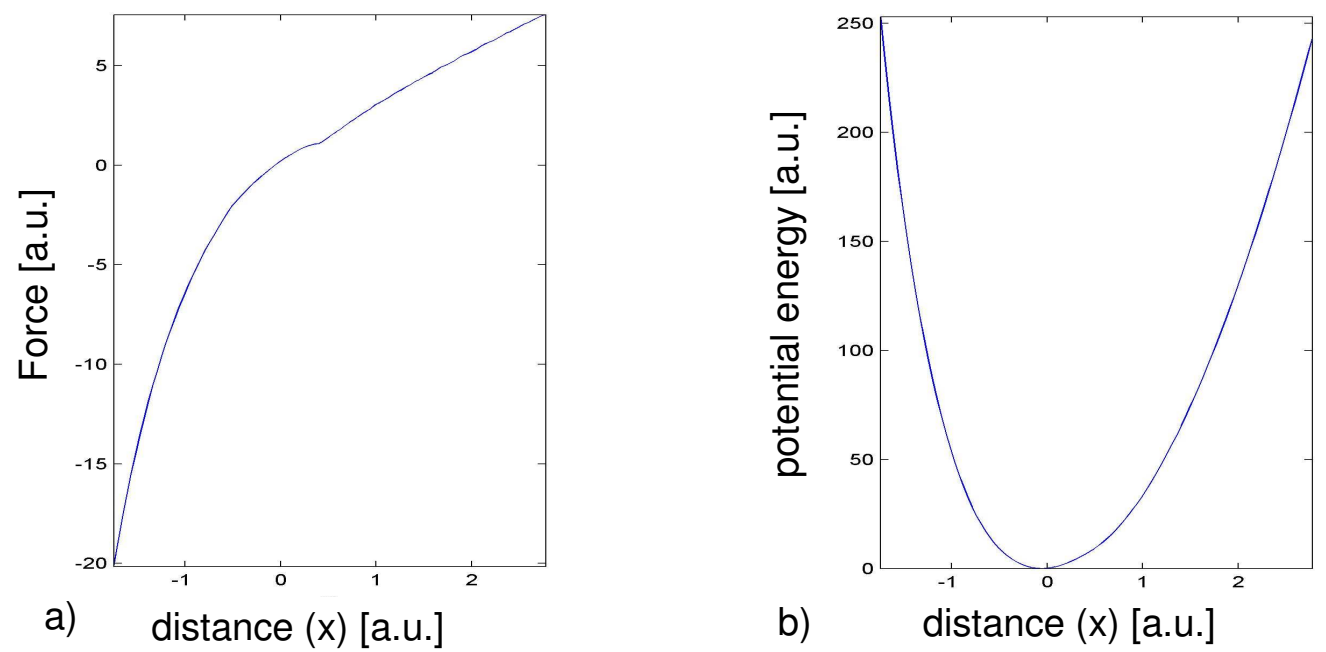

Figure 5: a) A plot of force vs. distance as determined from figure 1b, averaged over many oscillations. (b) Integrating over force vs. distance as a function of distance yields the shape of the original potential well. 